\title{
Preparation and Properties of Poly(L-lactide)-block-poly(trimethylene carbonate) as Biodegradable Thermoplastic Elastomer
}

\author{
Ji-Heung $\mathrm{KIM}^{\dagger}$ and Ju Hee LEE \\ Department of Chemical Engineering, Polymer Technology Institute, Sungkyunkwan University, \\ 300 Chunchun, Jangan, Suwon, Kyunggi 440-746, Korea
}

(Received November 8, 2001; Accepted January 16, 2002)

\begin{abstract}
Biodegradable ABA triblock copolymers of L-lactide and trimethylene carbonate with given compositions were prepared and chain-extended to produce high molecular weight polymer. The polymers were semicrystalline, and exhibited well microphase-segregated morphology with one crystalline poly(L-lactide) (PLLA) and the other soft and amorphous poly(trimethylene carbonate) (PTMC) block segments. The polymers could be cast into flexible and tough film, reversibly stretchable with elongation up to about $300 \%$. This material may provide a novel thermoplastic elastomer possessing desirable properties including biodegradability, biocompatibility and good mechanical properties including two-phase morphology. Also a preliminary result on the hydrolytic degradation behavior was discussed. KEY WORDS Poly(L-lactide) (PLLA)/ Poly(trimethylene carbonate) (PTMC) / Block Copolymer

/ Biodegradable / Thermoplastic Elastomer /
\end{abstract}

Modification of the properties of the brittle biodegradable polyesters such as polylactide (PLA), polyglycolide, and poly(hydroxy alkanoate) has been intensively investigated. ${ }^{1,2}$ Usually a biodegradable or biocompatible rubber is introduced to toughen the materials, so that the modified material remained biodegradable or biocompatible. PLA is one of the most intensively studied biodegradable synthetic polymer that has found significant applicability in the medical and pharmaceutical fields. ${ }^{3}$ Interest in PLA as a commercial thermoplastic has been increasing, and large-scale production has recently been announced. ${ }^{4}$ Though homo PLLA is limited by its inherent brittleness, its properties can be significantly enhanced and broadened by modification via copolymerization, which provides a number of advantages because the architecture and composition of the biomaterials can be tailored to control the material properties (by anionic or coordinated polymerization). In particular, block copolymerization may offer a broader spectrum of mechanical and degradation properties in order to meet the demands of various applications. Blocks with different physical properties, for example, one soft, amorphous and one hard, semicrystalline segment, can be utilized to modulate basic material behavior. ${ }^{5,6}$ For copolymers to be useful as thermoplastic elastomers (TPEs), the controlled synthesis of ABA triblock copolymer is often required. The mechanical properties of the polymer are enhanced by a microphase-separated morphology in which the soft phase gives elasticity and degradation, whereas the rigid phase provides mechanical strength and also acts as a physical crosslinker.

${ }^{\dagger}$ To whom correspondence should be addressed.
While ABA triblock copolymers containing polylactide segment and low $T_{\mathrm{g}}$ amorphous segment (e.g., polyisobutylene, polydimethyl-siloxane, and polyisoprene) have been reported, ${ }^{7-9}$ these systems have drawback in synthesis using specially prepared telechelic prepolymers and their inherent partial biodegradability. More recently, the synthesis and basic properties on the triblock copolymer systems containing poly(3-hydroxybutyrate), PHB, or poly(1,5-dioxepan2-one), poly(DXO), middle block has been investigated for the potential utilities as biodegradable thermoplastic elastomers. ${ }^{6,10}$ We were interested in using poly(trimethylene carbonate) (PTMC), a simple aliphatic polycarbonate, as soft and low- $T_{\mathrm{g}}$ segment. PTMC with the reported glass transition temperature of $-25^{\circ} \mathrm{C}$, is rubbery at room temperature and biodegradable both in vivo and in vitro, and hence, possesses potential applications in medical and environmental areas. ${ }^{11-13}$

Here we report the preparation and chain extension of P(LLA- $b$-TMC- $b$-LLA) triblock copolymers. Also the basic tensile properties as thermoplastic elastomer along with the phase-separated morphology will be discussed.

\section{EXPERIMENTAL}

\section{Chemicals and Measurements}

L-Lactide was purified by recrystallization twice from dry ethyl acetate and dried in vacuum at room temperature. Trimethylene carbonate (TMC) was purchased from Behringer Ingelheim and purified by sublimation. Diethylene glycol, hexamethylene diiso- 


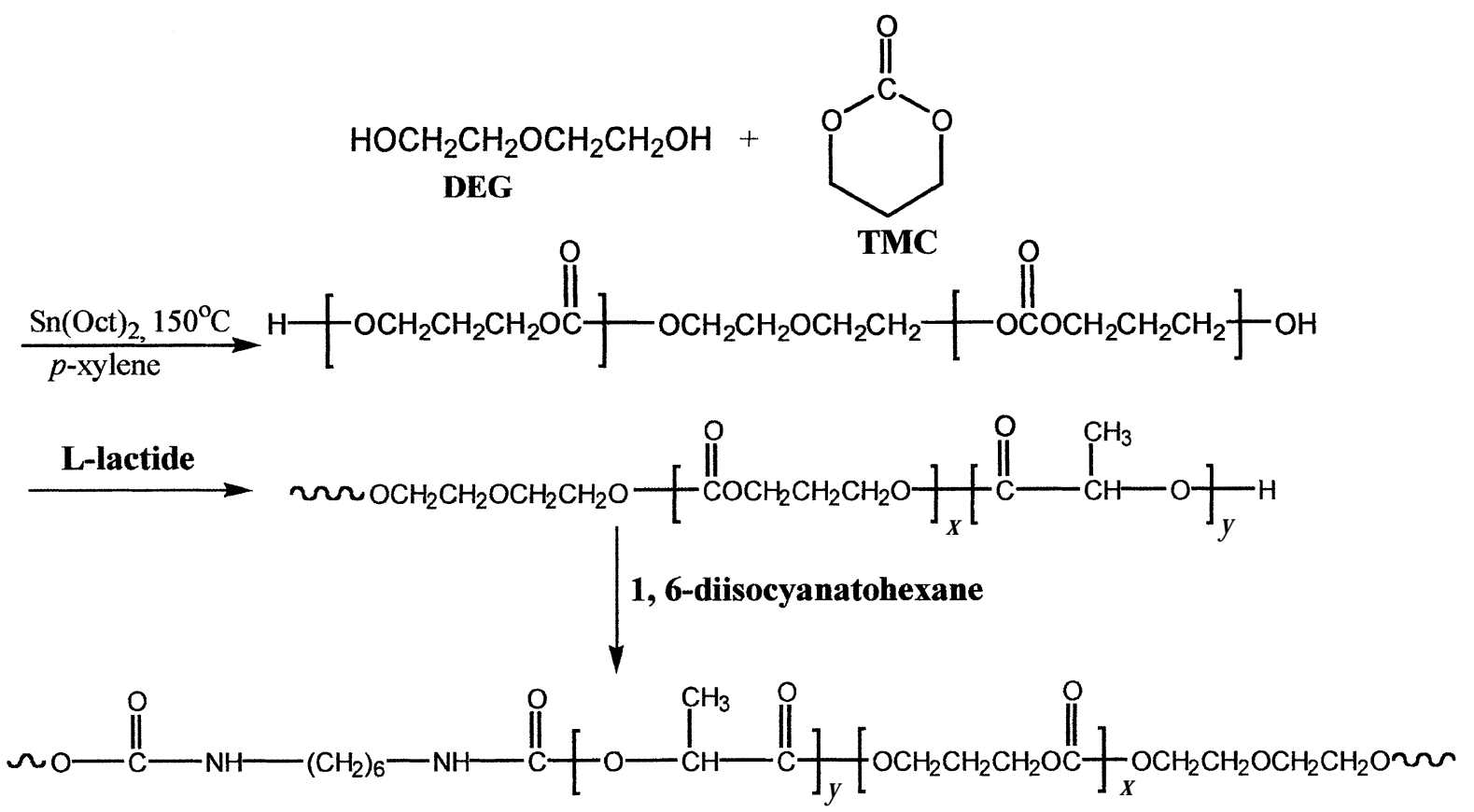

Scheme 1.

cyanate (HMDI) and stannous octoate was purchased from Aldrich Co. and used as received. $p$-Xylene was dried over calcium hydride $\left(\mathrm{CaH}_{2}\right)$ for $24 \mathrm{~h}$ prior to distillation.

Infrared spectra were obtained on a Unicam 1000 FTIR spectrometer. ${ }^{1} \mathrm{H}$ NMR spectra were taken on a Varian Unity Inova $500 \mathrm{MHz}$ Spectrometer using $\mathrm{CDCl}_{3}$ as the solvent. Thermal analysis was carried out on a Perkin-Elmer DSC/TGA7 Series thermal analysis system. The molecular weight data were obtained by gel permeation chromatography (GPC, Waters) using chlo-

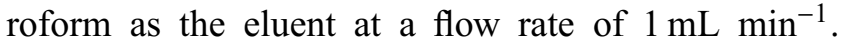
Polystyrene standards were used to calibrate the molecular weight. Tensile test was carried out using Instron model 5565, and the film morphology was observed by Optical Microscope (Samwon Scientific Ind. Co. LTD, Korea) model CSB-HA3.

Synthesis and Chain Extension of Triblock Copolymer, Poly ( $L A A-b-T M C-b-L L A)$

i) Triblock copolymers were prepared by sequential ring-opening polymerization of trimethylene carbonate and L-lactide in the presence of diethylene glycol as the typical polymerization procedure was described previously ${ }^{14}$ All glassware was frame-dried prior to use. The desired amounts of TMC ( $3 \mathrm{~g}, 29.4 \mathrm{mmol})$, diethylene glycol $(13 \mu \mathrm{L}, 0.5 \mathrm{~mol} \%$ of TMC) and catalyst ( $\mathrm{Sn}(\mathrm{oct})_{2}, 0.2 \mathrm{~mol} \%$ of total monomer) were weighed into a 3-necked round flask under a nitrogen atmosphere inside a drybox. The flask was fitted with a magnetic stirrer and condenser. $p$-Xylene $(15 \mathrm{~mL})$ was transferred to a flask using a syringe. The homoge- neous mixture was stirred for $24 \mathrm{~h}$ at $140^{\circ} \mathrm{C}$ and a small amount was sampled for analysis. The second monomer, L-lactide ( $4.23 \mathrm{~g}, 29.4 \mathrm{mmol})$, was charged into the reaction flask and stirred for additional $36 \mathrm{~h}$ to obtain a viscous polymer solution. The polymer formed was isolated by precipitation into a large amount of methanol/hexane, purified by reprecipitation from chloroform solution, washed, and finally dried in vacuum to obtain white and bulky powder.

ii) The block copolymer ( $1 \mathrm{~g})$, above prepared, was dissolved in $30 \mathrm{~mL}$ of dry toluene and heated to about $130^{\circ} \mathrm{C}$ to distilled off residual moisture inside as an azeotropic mixture. Then, a predetermined amount of HMDI, the chain-extending agent, was added by using microsyringe, and the reaction mixture was stirred for $4 \mathrm{~h}$ before precipitation into an excess amount of methanol. The resulting powder was filtered, washed, and dried in vacuum at $50^{\circ} \mathrm{C}$. To prepare film sample, the polymer was dissolved in chloroform, cast on a glass Petri-dish, solidified in air, and dried thoroughly in vacuo at $40^{\circ} \mathrm{C}$.

\section{RESULTS AND DISCUSSION}

\section{Synthesis of Triblock Copolymers and Their Chain- Extension}

As described in our previous communication, ${ }^{14}$ block copolymers were prepared by sequential ringopening polymerization of TMC and L-LA in the presence of small amount of diethyleneglycol (the whole reaction scheme including chain-extension is shown above), and the structure and basic thermal properties 
Table I. Characteristics of P(LLA- $b$-TMC- $b$-LLA) Triblock Copolymers

\begin{tabular}{|c|c|c|c|c|c|c|c|c|}
\hline & \multirow{2}{*}{$\frac{\text { Yield }^{\mathrm{a}}}{\%}$} & & \multicolumn{3}{|c|}{ GPC } & \multicolumn{2}{|c|}{ DSC } & \multirow{2}{*}{$\begin{array}{c}\text { TMC } \\
\text { Content }^{\mathrm{b}}\end{array}$} \\
\hline & & & $M_{\mathrm{n}}$ & $M_{\mathrm{w}}$ & $M_{\mathrm{w}} / M_{\mathrm{n}}$ & $\frac{T_{\mathrm{m}}}{\text { peak, }{ }^{\circ} \mathrm{C}}$ & $\frac{\Delta H_{\mathrm{m}}}{\mathrm{Jg}^{-1}}$ & \\
\hline \multirow{2}{*}{ CP1 } & \multirow{2}{*}{75} & PTMC & 6600 & 8700 & 1.32 & \multirow{2}{*}{139.4} & 16.4 & \multirow[b]{2}{*}{$43 \%$} \\
\hline & & Copolymer & 14200 & 21000 & 1.48 & & $(28.8)^{\mathrm{c}}$ & \\
\hline \multirow{2}{*}{$\mathrm{CP} 2$} & \multirow{2}{*}{80} & PTMC & 8500 & 11700 & 1.38 & \multirow{2}{*}{162.8} & 24.3 & \\
\hline & & Copolymer & 25200 & 43000 & 1.71 & & $(46.7)^{c}$ & $48 \%$ \\
\hline
\end{tabular}

${ }^{\mathrm{a}}$ Total copolymer yield. ${ }^{\mathrm{b}} \mathrm{TMC}$ unit $\mathrm{wt} \%$ in copolymer determined by ${ }^{1} \mathrm{H}$ NMR. ${ }^{\mathrm{c}}$ Calculated value based on PLA block weight.

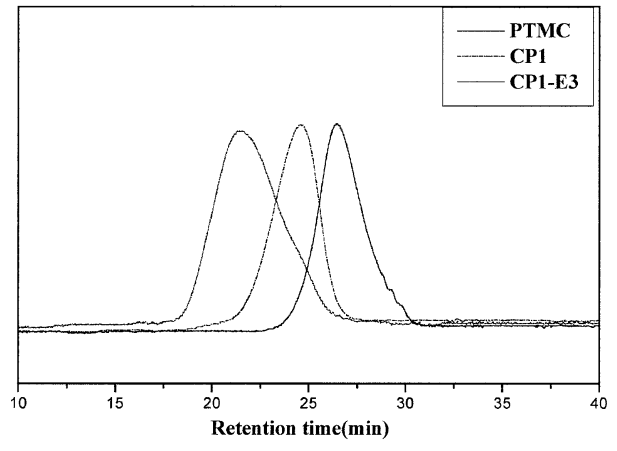

Figure 1. GPC chromatograms of PTMC prepolymer, triblock copolymer, and the chain-extended sample.

have been characterized by spectroscopy and thermal analysis. The characteristics of the prepared copolymers are shown in Table I. The molecular weight of the synthesized copolymers were $M_{\mathrm{n}} 14000$ and 25000, respectively, controllable by the amount of diethyleneglycol to a certain range. Attempts for the synthesis of much higher molecular weight materials were not successful and it seemed to be limited in our given polymerization reaction conditions. The resulting polymers could be cast into flexible films, though the sample was too weak to show a viable mechanical strength.

On the other hand, it is expected that the final triblock copolymers possess hydroxyl groups at both chain ends, which can be utilized for the chain extension process (or coupling reaction) to improve the molecular weight of as-polymerized materials. In this case, the chain-extended polymers should have multiblock chain structure, at least in part. Table II shows the results of chain extension for the copolymer CP1 using several different amount of diisocyanate compound (HMDI for this study). The chain-extended copolymer using HMDI with $[\mathrm{NCO}] /[\mathrm{OH}]$ ratio of 4 provided better result, giving 2.5-3 times of increase in the average molecular weight. Here, $[\mathrm{OH}]$ is the endhydroxyl concentration of triblock copolymer theoretically calculated from the $M_{\mathrm{n}}$ value by GPC. When the amount of HMDI was raised further, an additional increase in the molecular weight, however, was not observed. Instead, some amount of insoluble gel (3-4

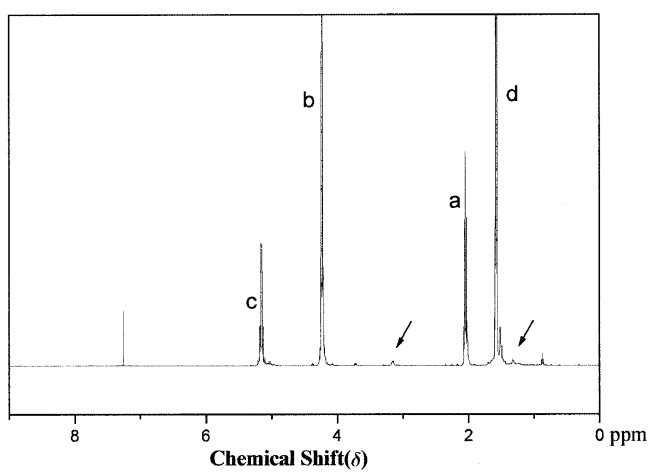

Figure 2. A typical ${ }^{1} \mathrm{H}$ NMR spectrum of chain-extended triblock copolymer.

$\mathrm{wt} \%$ ) was found to be formed in the reaction mixture, which is probably resulted from the crosslinking side reaction observed in the well-known urethane chemistry. Figure 1 shows the change in GPC chromatograms of PTMC prepolymer, triblock copolymer, and the chain-extended sample. First, a shift of distribution curve toward high molecular weight and unimodal distribution profile of both PTMC prepolymer and the corresponding copolymer suggested well formation of triblock copolymers from this synthesis. After chain extension, the distribution shifted further to higher molecular weight, though the molecular weight distribution became rather broader. In the similar manner, block copolymer $\mathbf{C P 2}$ was chain-extended by reacting with HMDI $([\mathrm{NCO}] /[\mathrm{OH}]=4)$, and a considerably high molecular weight polymer could be obtained with $M_{\mathrm{n}} 52000$, and $M_{\mathrm{w}} 135000$, respectively. The chain-extended polymers (CP1-E3 and CP2-E) were used to cast films to study the morphology and basic tensile properties described below.

A typical ${ }^{1} \mathrm{H}$ NMR spectrum of block copolymer after chain-extension is shown in Figure 2. The methine (c) and methyl protons (d) of the PLA block are shown at 5.17 and $1.5 \mathrm{ppm}$, and methylene protons $(\mathbf{a}, \mathbf{b})$ are shown at 2.06 and $4.24 \mathrm{ppm}$, respectively. Also methylene protons of HMDI moiety were observed at 3.13 and $1.34 \mathrm{ppm}$, respectively, to assure the incorporation of the unit in the copolymer structure. Monomer com- 
Table II. Result of Chain-Extension Using Several Different Amount of Diisocyanate Compound (HMDI)

\begin{tabular}{|c|c|c|c|c|c|c|c|c|}
\hline & \multirow{2}{*}[\mathrm{NCO}]{$/[\mathrm{OH}]^{\mathrm{a}}$} & \multirow{2}{*}{$\begin{array}{c}\text { Yield } \\
\%\end{array}$} & \multicolumn{3}{|c|}{ GPC } & \multicolumn{3}{|c|}{ DSC } \\
\hline & & & $M_{\mathrm{n}}$ & $M_{\mathrm{w}}$ & $M_{\mathrm{w}} / M_{\mathrm{n}}$ & $\frac{T_{\mathrm{g}}}{{ }^{\circ} \mathrm{C}}$ & $\frac{T_{\mathrm{m}}}{\text { peak, }{ }^{\circ} \mathrm{C}}$ & $\frac{\Delta H_{\mathrm{m}}}{\mathrm{Jg}^{-1}}$ \\
\hline $\mathrm{CP} 1$ & & & 14200 & 21000 & 1.48 & -8.2 & 139.4 & 16.4 \\
\hline CP1-E1 & 2 & 80 & 20900 & 34200 & 1.63 & -4.5 & 139.6 & 16.2 \\
\hline CP1-E2 & 3 & 85 & 26000 & 48500 & 1.82 & -2.1 & 131.0 & 15.3 \\
\hline CP1-E3 & 4 & 90 & 31800 & 59200 & 1.86 & -1.8 & 130.0 & 13.1 \\
\hline CP1-E4 & 5 & 92 & 27300 & 51300 & 1.88 & -2.7 & 129.5 & 12.3 \\
\hline
\end{tabular}

${ }^{\mathrm{a}}[\mathrm{OH}]$ in the ratio was theoretically calculated based on $M_{\mathrm{n}}$ of copolymer.

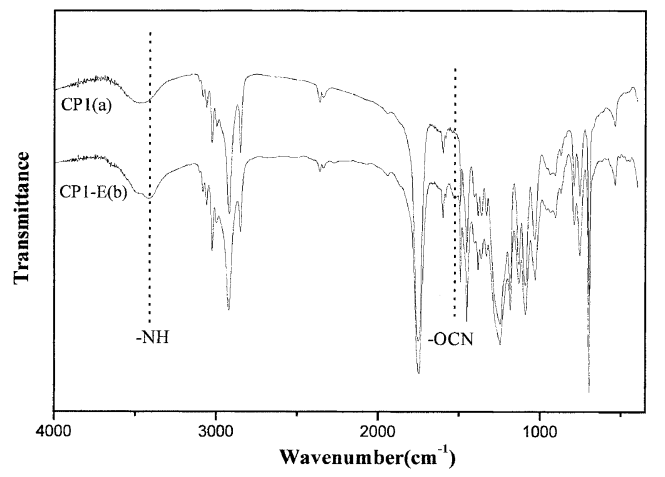

Figure 3. FT-IR spectra of triblock copolymer (a) and the chain-extended sample (b).

position of the copolymer was determined from the integration ratio between proton signals $\mathbf{c}$ and $\mathbf{a}$ (as the results are included in Table I).

Figure 3 depicts the FT-IR spectra of triblock copolymer and the chain-extended one. Copolymer $(\mathrm{CP} 1, \mathrm{a})$ has a strong $\mathrm{C}=\mathrm{O}$ stretching band at $1752 \mathrm{~cm}^{-1}$, and at 1185 and $1248 \mathrm{~cm}^{-1}$ of the $\mathrm{C}-\mathrm{C}-\mathrm{O}$ and $\mathrm{O}-\mathrm{C}-\mathrm{O}$ asymmetric stretching of the ester and the carbonate groups of the copolymer, respectively. From the chainextended products (CP1-E, b), additional characteristic absorption bands corresponding to $\mathrm{NH}$ stretching at $3420 \mathrm{~cm}^{-1}$, and $\mathrm{OCN}$ at $1510 \mathrm{~cm}^{-1}$ of urethane group were observed to identify the structures.

\section{Thermal Properties}

The thermal stability was measured by thermogravimetry in nitrogen. The triblock copolymers were stable to about $250^{\circ} \mathrm{C}$, with the decomposition onset shifting toward higher temperature after chainextension due to the much improved molecular weight of copolymer sample (Figure 4).

The glass transition and melting behavior of the materials were analyzed by low temperature differential scanning calorimetry. Typical thermograms (CP2) are shown in Figure 5 and the thermal properties of block copolymer CP2 and its chain-extended polymer CP2$\mathrm{E}$ (both powder and cast film) are summarized in Table III. The first heating scan (CP2) showed glass transition at around $-11^{\circ} \mathrm{C}$ and PLLA melting endotherm

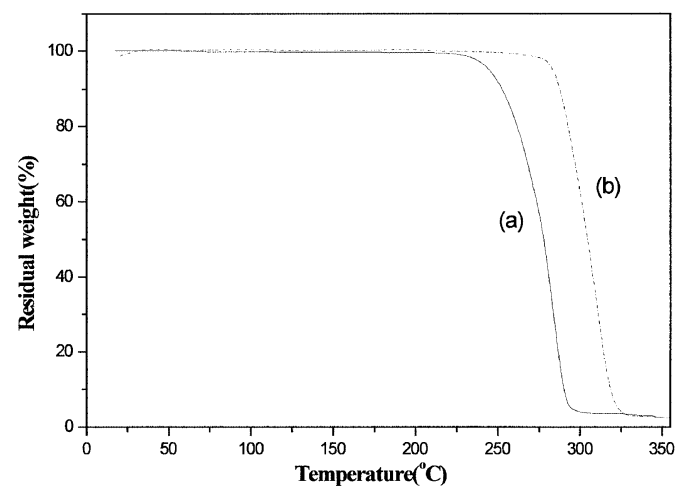

Figure 4. TGA thermograms of block copolymer (CP2, a) and the chain-extended one (CP2-E, b).

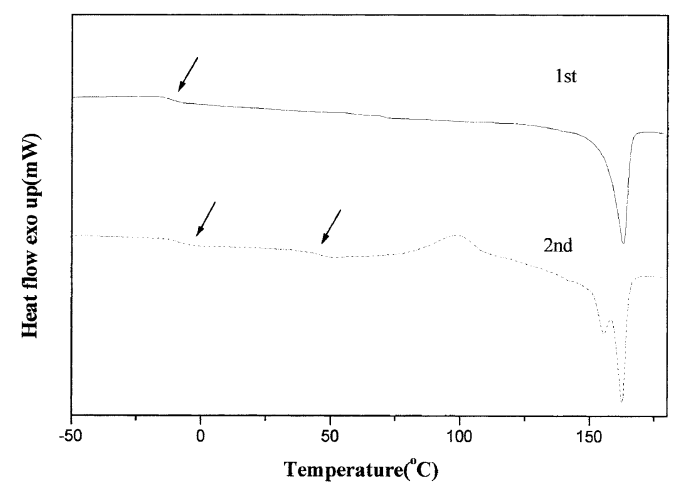

Figure 5. Typical DSC thermograms of triblock copolymer, CP2.

with the peak temperature of $163^{\circ} \mathrm{C}$, (and the following cooling scan showed a broad and weak crystallization exothermic transition at $110-80^{\circ} \mathrm{C}$ ). The second heating scan of the same sample showed two separate glass transitions at $c a .-9^{\circ} \mathrm{C}$ (corresponding to PTMC block), and at $c a .47^{\circ} \mathrm{C}$ (PLLA block), and also an exothermic transition at $90-110^{\circ} \mathrm{C}$ due to the cold crystallization, which is followed by melting transition again with peak temperature of $162^{\circ} \mathrm{C}$. The lower glass transition of PTMC block appeared at somewhat higher temperature than that of pure PTMC, which is probably resulted from the influence of high $T_{\mathrm{g}}$ PLLA block segment, and/or sorts of partial phase mixing between two polymers. Anyhow the glass transition temperature, observed at two different positions, and a rel- 
Table III. Thermal Properties of High M.W. Block Copolymer, Chain-Extended

\begin{tabular}{|c|c|c|c|c|c|c|c|c|c|}
\hline & \multicolumn{3}{|c|}{ DSC(1st) } & \multicolumn{6}{|c|}{ DSC(2nd) } \\
\hline & $\frac{T_{\mathrm{g}}}{{ }^{\circ} \mathrm{C}}$ & $\frac{T_{\mathrm{m}}}{\text { peak },^{\circ} \mathrm{C}}$ & $\frac{\Delta H_{\mathrm{m}}}{\mathrm{Jg}^{-1}}$ & $\frac{T_{\mathrm{g}_{1}}}{{ }^{\circ} \mathrm{C}}$ & $\frac{T_{\mathrm{g}_{2}}}{{ }^{\circ} \mathrm{C}}$ & $\frac{T_{\mathrm{m}}}{\text { peak, }{ }^{\circ} \mathrm{C}}$ & $\frac{\Delta H_{\mathrm{m}}}{\mathrm{Jg}^{-1}}$ & $\frac{T_{\mathrm{c}}}{\text { peak, }{ }^{\circ} \mathrm{C}}$ & $\frac{\Delta H_{\mathrm{c}}}{\mathrm{Jg}^{-1}}$ \\
\hline CP2 & -11.1 & 162.8 & 24.3 & -8.9 & 47.1 & 162.0 & 16.6 & 89.0 & -9.1 \\
\hline $\begin{array}{l}\text { CP2-E } \\
\text { (powder) }\end{array}$ & -9.9 & 159.0 & 18.9 & -7.2 & 38.0 & 159.3 & 14.8 & 106.0 & -8.2 \\
\hline $\begin{array}{l}\text { CP2-E } \\
\text { (cast film) }\end{array}$ & -10.3 & 158.7 & 15.6 & -8.4 & 36.5 & 158.9 & 14.5 & 100.6 & -8.0 \\
\hline
\end{tabular}

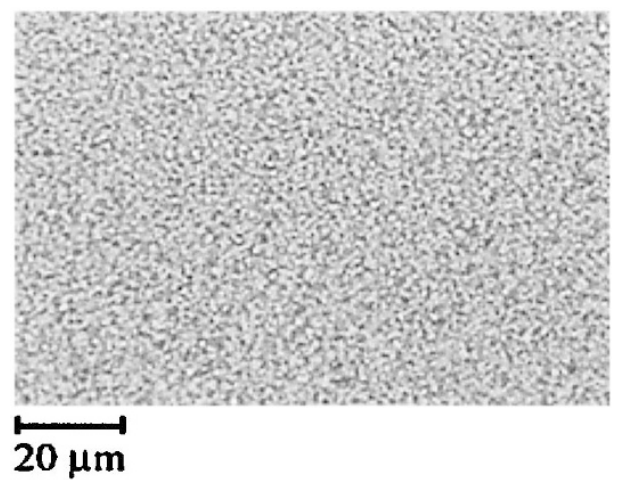

Figure 6. Optical micrograph of the cast polymer film (CP2E).

atively high crystallinity of the sample must suggest the phase-separated morphology of the block copolymers. The melting enthalpy $\left(\Delta H_{\mathrm{m}}\right)$ of the PLLA in triblock copolymer (CP2) was calculated to be 24.3 $\mathrm{Jg}^{-1}$. Based on the LLA content in the copolymer of $52 \mathrm{wt} \%$, the enthalpy can be expressed as 46.7 $\mathrm{J} \mathrm{g}^{-1}$-PLLA. This high value means not only high crystallinity of the resulting copolymer, but the crystallization of PLLA block is not hindered much by the PTMC block. (Also this result is obviously compared to the amorphous nature of random copolymer with similar monomer composition. ${ }^{15}$ ) The degree of crystallinity was calculated to be $c a$. $50 \%$ based on the theoretical pure PLLA, ${ }^{16}$ which is in the almost same ranges compared to those of homo PLLAs prepared by normal solution polymerization. ${ }^{17}$ This high crystallinity might be resulted from phase-segregated morphology of the prepared block copolymer, which enhances the crystallization of PLLA block by flexible and mobile PTMC segments with low $T_{\mathrm{g}}$. The chain-extended samples in both powder and film forms showed similar thermal properties, except a little decreases in melting temperature and the enthalpy values compared to those of sample before chain-extension. For the polymer sample CP1, the melting point and the crystallinity was relatively low, presumably due to the lower molecular weight of the material.

The suggested phase-separated morphology was observed by optical microscopy of the cast film. A rep-

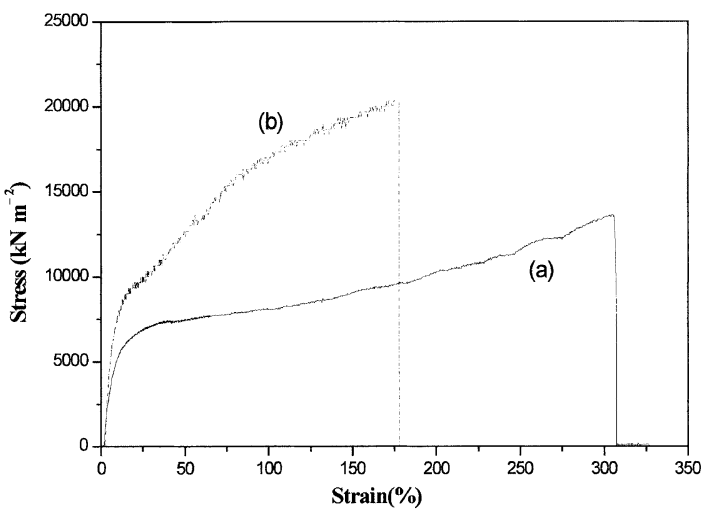

Figure 7. Stress-strain curve from Instron measurement; (a) CP1-E3, (b) CP2-E.

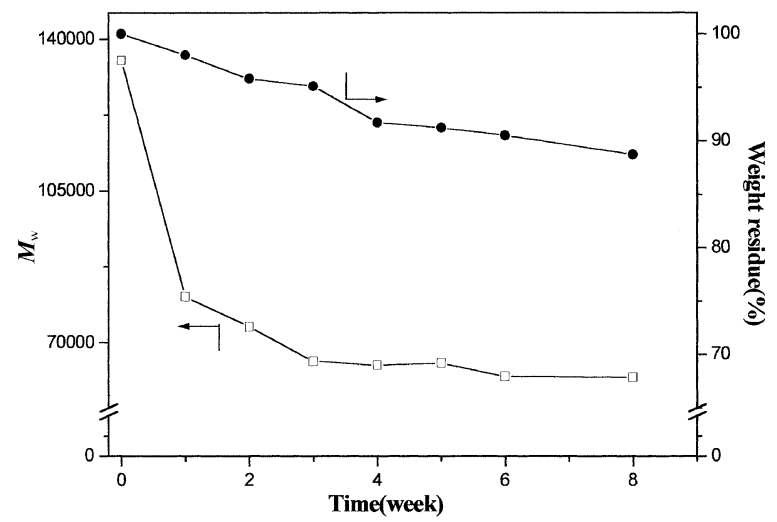

Figure 8. Weight and molar mass change of the cast films in saline phosphate buffer of $\mathrm{pH} 7.4$ (polymer sample: CP2-E).

resentative picture is shown in Figure 6. The interconnected, two-phase morphology comprising crystalline PLLA domain and amorphous PTMC-rich domain is apparent, and the domain size (width) was estimated to be about $1-2 \mu \mathrm{m}$. It is interesting that both the prepared ABA triblock copolymer and the chainextended one possess one crystalline block and another rubbery block component, forming well phaseseparated morphology. So, this material can exhibit behavior as a thermoplastic elastomer like other conventional TPEs known.

The tensile properties were measured by Instron, and a typical stress-strain curves are shown in Figure 7. The original translucent film was elongated to about 200- 
$300 \%$, variable by the different sample thickness and measurement condition. As the sample elongated, the film became opaque gradually by the condensed crystalline region, presumable accompanied by inducedcrystallization. When the force to stretched film was released, the sample contracted back toward initial state.

A preliminary degradation experiment of the cast film (about $30 \mu \mathrm{m}$ thick) was carried out in $\mathrm{pH} 7.4$ buffer saline solution at $40^{\circ} \mathrm{C}$. As the dipping time elapsed, the molecular weight dropped rapidly within a week to about half of original molecular weight, and then seemed to be leveled with a little decrease thereafter. The mass loss, however, was small with about $5 \mathrm{wt} \%$ in 2-3 wk, and additional 5-7 wt \% within the time period of $8 \mathrm{wk}$. The results were plotted in Figure 8 . It was expected that the block copolymer comprising with relatively hydrophobic monomers possess resistance to hydrolytic degradation. However, the chain-extended samples contains urethane linkage, which is more labile to the hydrolysis, so that the decreases in the molecular weight within a few weeks seems to be resulted from the preferable hydrolytic chain scission of the urethane bond. As an evident from the IR analysis, the sample after 4 wk did not show the characteristic absorption bands assignable to urethane linking groups.

To summarize, ABA triblock copolymers of Llactide and trimethylene carbonate with given compositions were prepared and chain-extended to produce high molecular weight polymer, forming elastomeric tough film. The polymers were partially crystalline and exhibited well phase-segregated morphology. This material may provide a novel thermoplastic elastomer possessing desirable properties including biodegradability, biocompatibility and good mechanical properties including elasticity. A preliminary result on the hydrolytic degradation behavior was also elucidated.
Acknowledgment. The present work was supported by the Korea Research Foundation Grant (KRF \# 20010547-200).

\section{REFERENCES}

1. H. Brandl, R. A. Gross, R. W. Lenz, and R. C. Fuller, "Advances in Biochemical Engineering/Biotechnology", T. K. Ghose and A. Fiechter, Eds., Springer-Verlag GmbH \& Co. KG, Berlin, 1990, vol. 41, p 77.

2. S. Matsumura, K. Tsukada, and K. Toshima, Macromolecules, 39, 3122 (1997).

3. E. S. Lipenski and R. G. Sinclair, Chem. Eng. Prog., 82, 26 (1986).

4. A. Tullo, Chemical and Engineering News, 78 (2000).

5. Y. K. Choi, Y. H. Bae, and S. W. Kim, Macromolecules, 31, 8766 (1998).

6. K. Stridsberg and A.-C. Albertsson, J. Polym. Sci., Part A: Polym. Chem., 38, 1774 (2000).

7. L. Sipos, M. Zsuga, and G. Deak, Macromol. Rapid Commun., 16, 935 (1995).

8. S. Zhang, Z. Hou, and K. E. Gonsalves, J. Polym. Sci., Part A: Polym. Chem., 34, 2737 (1996).

9. E. M. Frick and M. A. Hillmyer, Polym. Prepr., (Am. Chem. Soc., Div. Polym. Chem.), 42(1), 534 (2001).

10. S. Hiki, M. Miyamoto, and Y. Kimura, Polymer, 41, 7369 (2000).

11. A.-C. Albertsson and M. Eklund, J. Polym. Sci., Part A: Polym. Chem., 32, 265 (1994).

12. X. Chen, S. P. McCarthy, and R. A. Gross, Macromolecules, 30, 3470 (1997).

13. Y. Hori, Y. Gonda, Y. Takahashi, and T. Hagiwara, Macromolecules, 29, 804 (1996).

14. J.-H. Kim, S. Y. Lee, and D. J. Chung, Polym. J., 32, 1056 (2000).

15. E. Ruckenstein and Y. Yuan, J. Appl. Polym. Sci., 69, 1429 (1998).

16. S. Gogolewski and A. J. Pannings, J. Appl. Polym. Sci., 28, 1045 (1983).

17. W. J. Kim, S. Y. Lee, J.-H. Kim, S. H. Kim, and Y. H. Kim, J. Korean Ind. Eng. Chem., 10, 1028 (1999). 